\title{
Pelvic muscle floor rehabilitation as a therapeutic option in lifelong premature ejaculation: long-term outcomes
}

\author{
Antonio Luigi Pastore ${ }^{1,2}$, Giovanni Palleschi ${ }^{1,2}$, Andrea Fuschi ${ }^{1}$, Yazan Al Salhi ${ }^{1}$, Alessandro Zucchi, \\ Giorgio Bozzini ${ }^{4}$, Ester Illiano ${ }^{3}$, Elisabetta Costantini ${ }^{3}$, Antonio Carbone ${ }^{1,2}$
}

The aim of the study was to evaluate the long-term outcomes of pelvic floor muscle (PFM) rehabilitation in males with lifelong premature ejaculation (PE), using intravaginal ejaculatory latency time (IELT) and the self-report Premature Ejaculation Diagnostic Tool (PEDT) as primary outcomes. A total of 154 participants were retrospectively reviewed in this study, with 122 completing the training protocol. At baseline, all participants had an IELT $\leq 60 \mathrm{~s}$ and PEDT score $>11$. Participants completed a 12-week program of PFM rehabilitation, including physio-kinesiotherapy treatment, electrostimulation, and biofeedback, with three sessions per week, with $\mathbf{2 0 ~} \mathrm{min}$ for each component completed at each session. The effectiveness of intervention was evaluated by comparing the change in the geometric mean of IELT and PEDT values, from baseline, at 3, 6, and 12 months during the intervention, and at 24 and 36 months postintervention, using a paired sample 2-tailed $t$-test, including the associated $95 \%$ confidence intervals. Of the 122 participants who completed PFM rehabilitation, 111 gained control of their ejaculation reflex, with a mean IELT of $161.6 \mathrm{~s}$ and PEDT score of 2.3 at the 12-week endpoint of the intervention, representing an increase from baseline of $40.4 \mathrm{~s}$ and 17.0 scores, respectively, for IELT and PEDT $(P<0.0001)$. Of the 95 participants who completed the 36-month follow-up, $64 \%$ and $56 \%$ maintained satisfactory ejaculation control at 24 and 36 months postintervention, respectively.

Asian Journal of Andrology (2018) 20, 572-575; doi: 10.4103/aja.aja_30_18; published online: 3 July 2018

Keywords: biofeedback; electrostimulation; intravaginal ejaculatory latency time; pelvic floor rehabilitation; premature ejaculation; premature ejaculation diagnostic tool

\section{INTRODUCTION}

Premature ejaculation (PE) is a common sexual dysfunction in males which has a significant impact on the quality of life of the patients and partners. ${ }^{1,2}$ The role of the pelvic floor muscles in controlling the ejaculation is often overlooked in patients with $\mathrm{PE} .^{3}$

PE can either be lifelong or acquired. The 2014 updated criteria of the International Society for Sexual Medicine (ISSM) defines lifelong $\mathrm{PE}$ as ejaculation usually occurring within $1 \mathrm{~min}$ of vaginal penetration, and acquired $\mathrm{PE}$ as a clinically significant reduction in intravaginal ejaculatory latency time (IELT), usually in the range $\leq 3 \mathrm{~min}$ or less, inability to delay ejaculation and negative personal consequences, such as stress, bother, frustration, and/or the avoidance of sexual intimacy. ${ }^{4,5}$ Among factors contributing to acquired PE, IELT is defined as the time from vaginal intromission to intravaginal ejaculation ${ }^{4}$ and is often used as a parameter to quantify clinical response to therapy and as a standardized method to compare different treatment modalities in clinical trials.

In general, patients with $\mathrm{PE}$ are not aware of the role of pelvic floor muscles in the control of ejaculation. ${ }^{3,6}$ Therefore, it is possible that in many of these patients, PE may not result from an early arrival of the stimulus but, rather, due to a failure to selectively produce an effective contraction of the pelvic floor. ${ }^{7}$ Therefore, in our study, men with lifelong PE underwent pelvic floor muscle (PFM) rehabilitation, using a modification of techniques used for the treatment of urinary and fecal incontinence, ${ }^{6}$ and including physio-kinesiotherapy treatments, electrostimulation, and biofeedback.

The primary endpoint of our study was to evaluate the effectiveness of PFM rehabilitation by measuring the change, from baseline, in IELT and the self-administered Premature Ejaculation Diagnostic Tool (PEDT) over a follow-up period of 36 months. The secondary endpoint was to investigate the long-term effectiveness of PFM rehabilitation in training patients to recognize when and how to control the muscles involved in the ejaculatory reflex.

\section{PARTICIPANTS AND METHODS}

Participants

A total of 154 male participants were retrospectively reviewed in this study, which was conducted between September 2012 and December 2013. To be included in the study, participants had to be in a stable relationship, with the same partner, for at least 6 months and to engage 
in sexual intercourse $\geq 1$ per week. All participants provided written informed consent. Among the 154 participants enrolled into the study, $122(79.2 \%)$ completed the training protocol, with the other $32(20.8 \%)$ being excluded due to signs of prostatitis or personal preference to manage the problem with medication.

The study was conducted in accordance with the Ethical Principles for Medical Research Involving Human Subjects (World Medical Association, The Declaration of Helsinki Principles, 2000) and was approved by the local medical ethical committee of the hospital (ASL Lt/No. 43001/9674/2012).

PE was diagnosed by applying the ISSM definition of lifelong $\mathrm{PE},{ }^{5}$ with all participants having a baseline IELT of $\leq 60 \mathrm{~s}$ and PEDT test score $>11$. During the first visit, participants and their partners were interviewed individually and each was requested to provide an independent estimation of IELT. Participants also completed the PEDT, a self-administered questionnaire developed to standardize the diagnosis of PE and to capture the main elements of the DSM-IV-TR not considered by IELT (such as control, frequency, minimal sexual stimulation, distress, and interpersonal difficulty)..$^{8-10}$

All participants had previously tried different types of therapy, including esthetic creams, serotonergic antidepressants, and phosphodiesterase type 5 inhibitors (PDE5i), without a satisfactory response, especially with regard to adverse effects. Specifically, 47 participants had previously applied a local esthetic cream (EMLA $2.5 \mathrm{~g}$, AstraZeneca, Cambridge, UK) to the glans of the penis and penile shaft, $30 \mathrm{~min}$ before sexual intercourse, with no perceived significant benefit with regard to IELT, and with numbness of penis reported as the most frequent adverse event. Nineteen participants had been treated using tramadol hydrochloride, a narcotic-like pain reliever. Twenty-seven participants had been treated using selective serotonin reuptake inhibitor (SSRI) as needed, with nausea and cost of the drug being the main causes of treatment dropout. Another 19 participants were treated with PDE5i as needed (sildenafil $50 \mathrm{mg}$ or vardenafil $20 \mathrm{mg}$ ), $1 \mathrm{~h}$ to $3 \mathrm{~h}$ before sexual intercourse. All participants discontinued previous therapies at least 6 months before starting the program of PFM rehabilitation.

Preintervention IELT and PEDT were measured over a 4-week baseline period, with participants provided with a stopwatch and instructions on how to measure IELT and how to complete the PEDT. During this 4-week baseline period, participants were asked to have coitus at least four times. Couples were instructed not to use condoms or any topical esthetic cream and not to pause during intercourse or have interrupted intromission. Furthermore, participants were instructed to measure IELT only for the first intercourse, if intercourse took place more than once in a single session.

All participants underwent a physical examination prior to the start of the intervention. None of the participants had phimosis (19 patients were circumcised), frenulum breve, history of chronic prostatitis, or erectile dysfunction. The mean International Index of Erectile Function (IIEF) score of the study group prior to the intervention was 28.5 (95\% confidence interval [CI]: 26.2-28.7; standard deviation [s.d.] = 1.24). Participants also underwent a urological screening, which included the Meares-Stamey test, to exclude the presence of bacterial prostatitis, and a digital rectal examination. ${ }^{11}$

All participants completed the same PFM rehabilitation intervention, ${ }^{12,13}$ which consisted of the following components: physiokinesiotherapy to achieve a muscle contraction which provided participants with awareness of the motor activity; electrostimulation to the pudendal nerve for contraction of the puborectalis muscle, which causes the urethral sphincter to contract; and biofeedback to teach participants to control the muscle contractions of the perineal floor and the genitourinary sphincter. ${ }^{14,15}$ During the physiokinesiotherapy portion of the session, patients performed personalized physical exercises focused on isometric and isotonic contractions of the PFMs. Electrostimulation of the pudendal nerve, performed to enhance strengthening of the PFMs, was applied using a cylindrical anal probe, with contact between the electrodes in the anal probe and the anterior portion of the anal sphincter to stimulate the pubovisceral muscles (puborectal and pubourethral), with mild, painless electrical pulses used for stimulation..$^{14,15}$

Participants completed three 60 -min sessions per week, during which the three techniques were applied for a period of 20-min each. Outcome measures were obtained after the first 18 sessions ( 6 weeks) and then again at the end of the intervention (12 weeks).

Effectiveness of PFM rehabilitation was evaluated through comparison of the group mean IELT values and PEDT scores at the 3-, 6-, and 12-month time point of treatment, and at the 24- and 36-month time point of follow-up. A successful outcome of the intervention was defined as ability to control the ejaculatory reflex and a PEDT score $\leq 8.9,10$

\section{Statistical analyses}

The paired sample 2-tailed $t$-test, with the associated 95\% CI, was used to compare the geometric mean of IELTs and PEDT at each time point of measurement, with $P<0.05$ being considered to be statistically significant. Statistical analyses were conducted using SPSS (version 20.0, SPSS Inc., Chicago, IL, USA) and JMP (version 10.0, SAS Institute Cary, NC, USA).

\section{RESULTS}

The 122 participants included in the final analysis had a mean age of 24 years (range, 18-41 years), and a mean baseline IELT and PEDT of 40.4 s (95\% CI: 39.9-40.9) and 17.0 (95\% CI: 16.7-17.3), respectively. At the end of the 12-week PFM rehabilitation program, 111 (90.9\%) of the 122 patients reported a satisfactory ejaculation latency time from the start of intravaginal intercourse, with a mean IELT and PEDT of $161.6 \mathrm{~s}$ (95\% CI: 159.0-164.2) and 2.3 (95\% CI: 2.1-2.5), respectively (Table 1).

All participants were followed up for at least 2 years after the intervention, with 95 of the 122 participants $(77.8 \%)$ completing the follow-up to 36-month postintervention. The mean follow-up period was 36.4 months (range, 28.2-43.6 months), and satisfactory ejaculation latency time were maintained throughout the follow-up period (at least 2 years following the 12 -week intervention) in $64 \%$ of participants. Both

Table 1: Mean intravaginal ejaculatory latency time and premature ejaculation diagnostic tool scores and rate of satisfied patients after pelvic floor muscle rehabilitation. The paired sample two-tailed $t$-test with associated $95 \%$ confidence intervals was used to compare the geometric mean of intravaginal ejaculatory latency time and premature ejaculation diagnostic tool

\begin{tabular}{lcccc}
\hline & $\begin{array}{c}\text { Mean } \\
\text { IELT (s.d.) }\end{array}$ & $\begin{array}{c}\text { Mean } \\
\text { PEDT (s.d.) }\end{array}$ & $\begin{array}{c}\text { Patient } \\
\text { evaluated (n) }\end{array}$ & $\begin{array}{c}\text { Satisfied } \\
\text { patient (\%) }\end{array}$ \\
\hline Baseline & $40.4(8.7)$ & $17.0(2.2)$ & 122 & \\
At 3 months & $161.6(14.5)$ & $2.3(1.1)$ & 122 & 90.2 \\
At 6 months & $157.7(12.9)$ & $2.8(1.3)$ & 122 & 85.3 \\
At 12 months & $156.3(13.1)$ & $3.4(1.6)$ & 122 & 78.5 \\
At 24 months & $149.0(12.2)$ & $4.6(1.0)$ & 122 & 63.9 \\
At 36 months & $138.5(10.5)$ & $5.7(1.2)$ & 95 & 56.8 \\
$P$ & $<0.0001$ & $<0.0001$ & & \\
\hline IELT: intravaginal ejaculatory latency time; PEDT: premature ejaculation diagnostic tool; \\
s.d.: standard deviation
\end{tabular}

IELT: intravaginal ejaculatory latency time; PEDT: premature ejaculation diagnostic tool s.d.: standard deviation 
IELT and PEDT were improved at the endpoint of follow-up, compared to baseline (IELT, $P<0.0001$; Table 1 and Figure 1; PEDT, $P<0.0001$; Table 1 and Figure 2). Compared to baseline, IELT improved by 40.4 $s$ and the PEDT by a score of $17.04(P<0.0001)$.

Of note, at the 3-month time point of measurement, $11(9.0 \%)$ participants did not report any significant improvement in ejaculatory latency time. At the 2-year time point of follow-up, 27 (22.1\%) of the 122 patients who reported a significant improvement in clinical condition after PFM rehabilitation voluntarily dropped out of the study. At 24- and 36-month time point of follow-up, $63.9 \%$ and $56.8 \%$ of participants, respectively, maintained satisfactory ejaculation control (Table 1). No adverse effect was reported that participants could have led to the discontinuation of the PFM rehabilitation.

\section{DISCUSSION}

Currently, the recommended management of PE includes behavioral and psychotherapy, drug therapy, and/or a combination of these. ${ }^{16-19}$ Pharmacotherapy, such as dapoxetine (a short-acting SSRI that is the only approved pharmacological treatment for premature ejaculation), used as necessary, or other off-label antidepressants (daily SSRIs), which require regular dosage, have the highest level of evidence of effectiveness. ${ }^{16-19}$ Various other forms of pharmacotherapy have been used for the treatment of PE, including topical local anesthetics, tramadol, PDE5 inhibitors, and $\alpha$-adrenergic blockers, each being supported by varying levels of clinical evidence. ${ }^{16-19}$ Research evidence has supported a possible active role of the PFMs, particularly the ischiocavernosus and bulbocavernosus muscles and sphincters, in the control of ejaculation, as confirmed by a significant increase in electromyography activity during the entire physiological process. ${ }^{20}$ As we have previously reported, ${ }^{15,21}$ physiokinesiotherapy and electrostimulation are designed to improve the contractile strength of the perineal muscles, whereas biofeedback is used to facilitate patients' learning to recognize and contract PFMs to increase the closing strength of the urethral sphincter. However, PFM training does require a few months for a patient to understand the dynamics of the sequence of events, learn to control the ejaculatory reflex and naturally apply this learning during sexual intercourse. ${ }^{11,21}$ In general, twenty sessions of training are required for a patient to gain sufficient control over the ejaculatory reflex. It is reasonable to assume that some patients may require a faster therapeutic approach, such as drug therapy. Another limitation of training is that not all patients are able to perform a selective control of the pelvic floor muscles or recognize the sensation that precedes the inevitability of ejaculatory reflex. However, when PFM rehabilitation is performed adequately, patients could benefit from PE control for 6 to 36 months, without the use of drugs. ${ }^{21}$ The findings of the current study confirm our previous hypothesis regarding the clinical benefit of PFM rehabilitation in allowing patients to achieve pelvic floor awareness and improved self-confidence and sense of control over their ejaculatory reflex. ${ }^{21}$ In the current study,

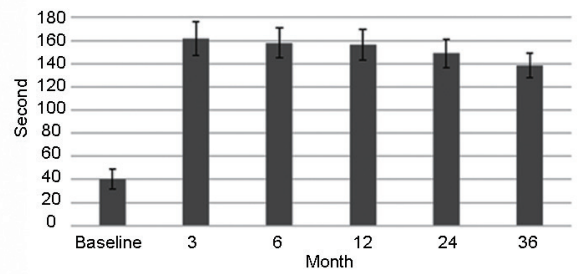

Figure 1: IELTs evaluated at 3, 6, 12, 24, and 36 months after PFM rehabilitation. IELT: intravaginal ejaculatory latency time; PFM: pelvic floor muscle. the improvement in IELT, from baseline, of $146.2 \pm 38.3 \mathrm{~s}$ was greater after 12 weeks of training than in our previous study. ${ }^{21}$ Furthermore, compared to baseline, IELT and PEDT were found to be significantly improved among those patients who completed the follow-up to $24(63.9 \%)$ and $36(56.8 \%)$ months postintervention. These results represent another important achievement obtained by way of an easily learned technique that can be mastered using pelvic floor biofeedback. Moreover, no adverse effects of the PFM rehabilitation protocol were identified, compared to other medical therapies such as gastrointestinal symptoms (nausea and diarrhea) and dizziness and headaches, which have been associated with the use of dapoxetine. ${ }^{22,23}$ In addition, the long-term effects of dapoxetine on reproductive functions should be cleared as some recent data demonstrated its negative impact on fertility. ${ }^{24}$

To the best of our knowledge, the present study has the longest follow-up period (36 months) reported to date for any intervention for PE. However, several limitations of our study should be noted. The main limitation regards the lack of a control group. It is important to note that we planned our study without a control arm as we consider that it is unethical to not treat patients with $\mathrm{PE}$ when it impacts quality of life. We also used the PEDT test as a primary outcome measure, although the Italian version of the PEDT remains to be validated. Furthermore, we used the PEDT as a measure of the impact of PFM training on $\mathrm{PE}$, noting that the PEDT was designed to screen for PE rather than as patient-reported outcome for an intervention. In addition, we did not consider the perception of orgasmic intensity in our study, which has previously been reported in men with PE. ${ }^{25}$ Future studies should consider using the validated "Orgasmometer," which is a psychometric tool that evaluates the subjective perception of orgasmic intensity ${ }^{25}$ on a 10-point scale. There is a lack of standardized measures to assess improvement following the PFM intervention, with the treatment parameters difficult to define as they are largely based on sensory perception. Nonetheless, there is a continued need for evidence-based research to validate the role of physiokinesiotherapy in the treatment of PE. Although the type, the amount, and the focus (relaxation, strength, support, or control) of exercise required have not yet been standardized, the long-term results obtained in our patients with lifelong PE suggest that it may be considered as a valid therapeutic option for patients with $\mathrm{PE}$.

\section{CONCLUSION}

The pelvic floor muscle rehabilitation protocol is easy to perform, with no reported adverse effects. Although it has not yet been standardized, the long-term results obtained in our patients with lifelong premature ejaculation suggest that it may be considered as a therapeutic option for patients with premature ejaculation. However, controlled trials are needed before making final evaluation on the effectiveness of pelvic floor muscle rehabilitation in premature ejaculation patients.

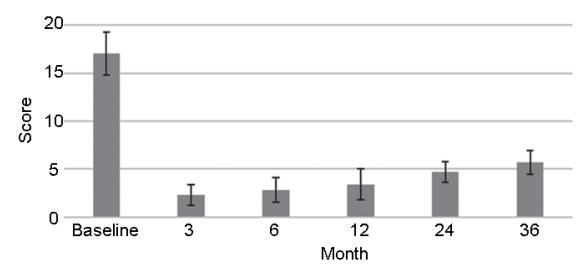

Figure 2: PEDT scores evaluated at 3, 6, 12, 24, and 36 months after PFM rehabilitation. PEDT: premature ejaculation diagnostic tool; PFM: pelvic floor muscle. 


\section{AUTHOR CONTRIBUTIONS}

ALP, GP, YAS, AF, GB, EI, AZ: Study conception and design, data collection and statistical analyses; ALP, GP, YAS, AF, AC; EC: Writing and revision of the manuscript.

\section{COMPETING INTERESTS}

All authors declared no competing interests.

\section{REFERENCES}

1 Saitz TR, Serefoglu EC. The epidemiology of premature ejaculation. Trans/ Androl Urol 2016; 5: 409-15.

2 Jannini EA, Lenzi A. Epidemiology of premature ejaculation. Curr Opin Urol 2005; 15: 399-403.

3 La Pera G. Awareness of the role of the pelvic floor muscles in controlling the ejaculatory reflex: preliminary results. Arch Ital Urol Androl 2012; 84: 74-8.

4 Waldinger MD, Quinn P, Dilleen M, Mundayat R, Schweitzer DH, et al. A multinational population survey of intravaginal ejaculation latency time. J Sex Med 2005; 2: 492-7.

5 Serefoglu EC, McMahon CG, Waldinger MD, Althof SE, Shindel A, et al. An evidence-based unified definition of lifelong and acquired premature ejaculation: report of the second international society for sexual medicine ad hoc committee for the definition of premature ejaculation. Sex Med 2014; 2: 41-59.

6 Siegel AL. Pelvic floor muscle training in males: practical applications. Urology 2014; 84: 1-7.

7 Serefoglu EC, Saitz TR, Trost L, Hellstrom WJ. Premature ejaculation: do we have effective therapy? Transl Androl Urol 2013; 2: 45-53.

$8 \mathrm{Kam}$ SC, Han DH, Lee SW. The diagnostic value of the premature ejaculation diagnostic tool and its association with intravaginal ejaculatory latency time. J Sex Med 2011; 8: 865-71.

9 Symonds T, Perelman MA, Althof S, Giuliano F, Martin M, et al. Development and validation of a premature ejaculation diagnostic tool. Eur Urol 2007; 52: 565-73.

10 Symonds T, Perelman MA, Althof S, Giuliano F, Martin M, et al. Further evidence of the reliability and validity of the premature ejaculation diagnostic tool. Int J Impot Res 2007; 19: 521-5.

11 Screponi E, Carosa E, Di Stasi SM, Pepe M, Carruba G, et al. Prevalence of chronic prostatitis in men with premature ejaculation. Urology 2001; 58: 198-202.

12 La Pera G. Awareness and timing of pelvic floor muscle contraction, pelvic exercises and rehabilitation of pelvic floor in lifelong premature ejaculation: 5 years experience. Arch Ital Urol Androl 2014; 86: 123-7.

13 Hay-Smith J, Herbison P, Mørkved S. Physical therapies for prevention of urinary and faecal incontinence in adults. Cochrane Database Syst Rev 2007; 4: CD003191.

14 La Pera G, Nicastro A. A new treatment for premature ejaculation. The rehabilitation of the pelvic floor. J Sex Marital Ther 1996; 22: 22-6.

15 Pastore AL, Palleschi G, Leto A, Pacini L, lori F, et al. A prospective randomized study to compare pelvic floor rehabilitation and dapoxetine for treatment of lifelong premature ejaculation. Int J Androl 2012; 35: 528-33.

16 Althof SE, McMahon CG, Waldinger MD, Serefoglu EC, Shindel AW, et al. An update of the International Society of Sexual Medicine's guidelines for the diagnosis and treatment of premature ejaculation (PE). J Sex Med 2014; 11: 1392-422.

17 Jannini EA, Ciocca G, Limoncin E, Mollaioli D, Di Sante S, et al. remature ejaculation: old story, new insights. Fertil Steril 2015; 104: 1061-73.

18 Clement P, Giuliano F. Physiology and pharmacology of ejaculation. Basic Clin Pharmacol Toxicol 2016; 119: 18-25.

19 Giuliano F, Clèment P. Pharmacology for the treatment of premature ejaculation. Pharmacol Rev 2012; 64: 621-44.

20 Pischedda A, Fusco F, Curreli A, Grimaldi G, Pirozzi Farina F. Pelvic floor and sexual male dysfunction. Arch Ital Urol Androl 2013; 85: 1-7.

21 Pastore AL, Palleschi G, Fuschi A, Maggioni C, Rago R, et al. Pelvic floor muscle rehabilitation for patients with lifelong premature ejaculation: a novel therapeutic approach. Ther Adv Urol 2014; 6: 83-8.

22 Kaufman JM, Rosen RC, Mudumbi RV, Tesfaye F, Hashmonay R, et al. Treatment benefit of dapoxetine for premature ejaculation: results from a placebo-controlled phase III trial. BJU Int 2009; 103: 651-8.

23 Russo A, Capogrosso P, Ventimiglia E, La Croce G, Boeri L, et al. Efficacy and safety of dapoxetine in treatment of premature ejaculation: an evidence-based review. Int J Clin Pract 2016; 70: 723-33.

24 El Mazoudy R, Abdel Hameed N, El Masry A. Paternal dapoxetine administration induced deterioration in reproductive performance, fetal outcome, sexual behaviour and biochemistry of male rats. Int J Impot Res 2015; 27: 206-14.

25 Limoncin E, Lotti F, Rossi M, Maseroli E, Gravina GL, et al. The impact of premature ejaculation on the subjective perception of orgasmic intensity: validation and standardisation of the 'Orgasmometer'. Andrology 2016; 4: 921-6.

This is an open access journal, and articles are distributed under the terms of the Creative Commons Attribution-NonCommercial-ShareAlike 4.0 License, which allows others to remix, tweak, and build upon the work non-commercially, as long as appropriate credit is given and the new creations are licensed under the identical terms.

(C)The Author(s)(2018) 